\title{
The Effect of Treadmill Exercise on Gait Efficiency During Overground Walking in Adults With Cerebral Palsy
}

\author{
On-Yoo Kim, $\mathrm{MD}^{1}$, Yoon-Kyum Shin, $\mathrm{MS}^{2}$, Young Kwon Yoon, $\mathrm{MD}^{3}$, \\ Eu Jeong Ko, $\mathrm{MD}^{1}$, Sung-Rae Cho, $\mathrm{MD}, \mathrm{PhD}^{1,2,4,5}$
}

\begin{abstract}
${ }^{1}$ Department of Rehabilitation Medicine and Research Institute of Rehabilitation Medicine, ${ }^{2}$ Brain Korea 21 PLUS Project for Medical Science, ${ }^{3}$ Graduate School of Medical Science, ${ }^{4}$ Avison Biomedical Research Center, and ${ }^{5}$ Rehabilitation Institute of Neuromuscular Disease, Yonsei University College of Medicine, Seoul, Korea
\end{abstract}

Objective To investigate the effect of treadmill walking exercise as a treatment method to improve gait efficiency in adults with cerebral palsy (CP) and to determine gait efficiency during overground walking after the treadmill walking exercise.

Methods Fourteen adults with CP were recruited in the experimental group of treadmill walking exercise. A control group of 7 adults with CP who attended conventional physical therapy were also recruited. The treadmill walking exercise protocol consisted of 3-5 training sessions per week for 1-2 months (total 20 sessions). Gait distance, velocity, $\mathrm{VO}_{2}, \mathrm{VCO}_{2}, \mathrm{O}_{2}$ rate $(\mathrm{mL} / \mathrm{kg} \cdot \mathrm{min})$, and $\mathrm{O}_{2}$ cost $(\mathrm{mL} / \mathrm{kg} \cdot \mathrm{m})$ were assessed at the beginning and at the end of the treadmill walking exercise. The parameters were measured by KB1-C oximeter.

Results After the treadmill walking exercise, gait distance during overground walking up to 6 minutes significantly increased from $151.29 \pm 91.79$ to $193.93 \pm 79.01 \mathrm{~m}$, and gait velocity increased from $28.09 \pm 14.29$ to $33.49 \pm 12.69 \mathrm{~m} /$ $\min (\mathrm{p}<0.05)$. Energy efficiency evaluated by $\mathrm{O}_{2}$ cost during overground walking significantly improved from $0.56 \pm 0.36$ to $0.41 \pm 0.18 \mathrm{~mL} / \mathrm{kg} \cdot \mathrm{m}(\mathrm{p}<0.05)$, whereas $\mathrm{O}_{2}$ rate did not improve significantly after the treadmill walking exercise. On the other hand, gait velocity and $\mathrm{O}_{2}$ cost during overground walking were not significantly changed in the control group.

Conclusion Treadmill walking exercise improved the gait efficiency by decreased energy expenditure during overground walking in adults with CP. Therefore, treadmill walking exercise can be an important method for gait training in adults with $\mathrm{CP}$ who have higher energy expenditure.

Keywords Cerebral palsy, Exercise, Walking, Gait, Efficiency

Received July 1, 2014; Accepted September 11, 2014

Corresponding author: Sung-Rae Cho

Department of Rehabilitation Medicine and Research Institute of Rehabilitation Medicine, Yonsei University College of Medicine, 50 Yonsei-ro, Seodaemun-gu, Seoul 120-749, Korea

Tel: +82-2-2228-3715, Fax: +82-2-363-2795, E-mail: srcho918@yuhs.ac

(c) This is an open-access article distributed under the terms of the Creative Commons Attribution Non-Commercial License (http://creativecommons.org/ licenses/by-nc/3.0) which permits unrestricted noncommercial use, distribution, and reproduction in any medium, provided the original work is properly cited. Copyright $\odot 2015$ by Korean Academy of Rehabilitation Medicine 


\section{INTRODUCTION}

Cerebral palsy (CP) describes a group of disorders in movement and posture that limits activity and is attributed to disturbances in the developing fetal or infant brain [1]. Treadmill walking exercise may improve walking activity in children with $\mathrm{CP}$, as it provides an opportunity to repetitively train the entire gait cycle and facilitate an improved gait pattern [2]. Treadmill walking exercise has been associated with increases in strength and decreases in energy expenditure [3]. Moreover, partial body-weight supported treadmill walking exercise to reinforce the pattern of walking achieved on the treadmill has been used to force an increase in walking speed and distance [4]. Several preliminary studies suggest partial body-weight supported treadmill walking exercise as effective in improving walking among ambulatory children with CP [2-5] and post-stroke locomotion recovery $[6,7]$; however, there are few studies on ambulatory adults with $\mathrm{CP}$ without a body-weight support. Therefore, more research is needed to determine whether treadmill walking exercise is effective for adult CP patients who usually show decline in walking activity and aerobic capacity $[2,3,5]$.

Oxygen consumption $\left(\mathrm{VO}_{2}\right)$ is considered as the gold standard for assessing energy expenditure during an activity [8]. Walking energy consumption is a valid evaluation of walking performance in individuals. Gait disabilities, such as CP and stroke, increase the energy expenditure of walking and the intensity of physical effort with altered walking speeds [9]. Our study was designed to evaluate the effect of a treadmill walking exercise as a mean of improving the energy expenditure of walking and gait efficiency in adults with CP. We also determined improvement in parameters such as gait speed, distance and energy consumption during overground walking or treadmill walking in response to such exercise in ambulatory $\mathrm{CP}$ patients.

\section{MATERIALS AND METHODS}

\section{Participants}

Fourteen ambulatory adults with CP (7 male and 7 female; mean age of 28 years) were recruited to the treadmill walking exercise experimental group. A control group of 7 adults with $\mathrm{CP}$, who attended conventional physical therapy alone, were also recruited. This study was approved by the Institutional Review Board (IRB No. 4-2008-0624). Total 21 CP participants were randomly allocated to either the experimental or control group at a 2:1 ratio, using a central telephone randomization service [10]. Inclusion criteria were as follows: the ability to ambulate independently indoors without a gait aid and the ability to follow verbal directions for standardized testing. The following were used as exclusion criteria: orthopedic surgery such as musculotendinous lengthening and femoral derotation osteotomy, neurosurgery such as selective dorsal rhizotomy and intrathecal baclofen pump implantation, or botulinum toxin injection into gastrocnemius, medial hamstring and hip adductors in the past 12 months, and clinically evident unstable cardiac status, or any premorbid conditions. All participants in the experimental group performed a treadmill walking exercise for 30 minutes and conventional physical therapy. The treadmill walking exercise protocol for the current study consisted of 3-5 training sessions per week for 1-2 months (total 20 sessions). Participants in the control group received conventional physical therapy in the same sessions.

\section{Six-minute walking test}

The 6-minute walk is a reliable and valid measure of walking endurance. The instructions for the test are standardized according to Lipkin et al. [11]. For the 6-minute walking test during overground walking, the patients were encouraged to walk as fast as possible while not running, and the total walking distance was recorded. If the patients could not maintain overground walking for 6 minutes, they finished the walking endurance test, and the distance walked until the time was measured.

\section{Measurement of energy expenditure}

Energy expenditure was examined during treadmill walking at a self-selected comfortable speed and overground walking on the first and the last day of treadmill walking exercise. The energy expenditure was measured using KB1-C oximeter (AeroSport Inc., Ann Arbor, MI, USA). Oxygen consumption of walking is the volume of oxygen consumed per $\mathrm{kg}$ of body weight for a given time. Oxygen rate of the experimental subjects using a breathing mask were analyzed with oxygen gas analyzer built into the computer. Oxygen uptake per minute $\left(\mathrm{VO}_{2}, \mathrm{~mL} /\right.$ min), carbon dioxide production amount per minute 
$\left(\mathrm{VCO}_{2}, \mathrm{~mL} / \mathrm{min}\right)$, respiratory volume per minute (VE, L/ $\mathrm{min})$, oxygen consumption rate $\left(\mathrm{O}_{2}\right.$ rate, $\left.\mathrm{mL} / \mathrm{kg} \cdot \mathrm{min}\right)$, and oxygen consumption cost $\left(\mathrm{O}_{2}\right.$ cost, $\left.\mathrm{mL} / \mathrm{kg} \cdot \mathrm{m}\right)$ were measured. The $\mathrm{O}_{2}$ cost was determined by dividing $\mathrm{O}_{2}$ consumption by the velocity of walking, as an indicator of the gait efficiency [9].

\section{Statistical analysis}

Wilcoxon signed-rank test for paired samples before and after treadmill walking exercise was used to compare the parameters of energy consumption test during treadmill walking or overground walking. Additionally, MannWhitney U test was used to compare the parameters of

Table 1. General characteristics of the subjects

\begin{tabular}{lcc}
\hline & $\begin{array}{c}\text { Experimental } \\
\text { group }(\mathbf{n = 1 4})\end{array}$ & $\begin{array}{c}\text { Control } \\
\text { group (n=7) }\end{array}$ \\
\hline Age (yr) & $28.64 \pm 8.55$ & $24.43 \pm 9.07$ \\
Sex (male:female) & $7: 7$ & $4: 3$ \\
Height $(\mathrm{cm})$ & $159.62 \pm 7.32$ & $153.29 \pm 14.86$ \\
Weight $(\mathrm{kg})$ & $54.07 \pm 10.96$ & $55.0 \pm 17.83$ \\
BMI $\left(\mathrm{kg} / \mathrm{m}^{2}\right)$ & $20.66 \pm 2.88$ & $21.58 \pm 3.77$ \\
\hline Systolic BP $(\mathrm{mmHg})$ & $121.85 \pm 13.38$ & $114.71 \pm 22.79$ \\
Diastolic BP (mmHg) & $77.85 \pm 8.32$ & $72.29 \pm 14.38$ \\
\hline
\end{tabular}

Values are presented as mean \pm standard deviation or number of subjects.

Experimental group, treadmill walking exercise and conventional physical therapy; control group, conventional physical therapy alone; BMI, body mass index; BP, blood pressure.
$\Delta$ distance, $\Delta$ velocity, $\Delta \mathrm{O}_{2}$ rate, and $\Delta \mathrm{O}_{2}$ cost calculated by the subtraction from post-treatment to pre-treatment values between experimental and control groups. All statistical analyses were performed using SPSS ver. 20.0 (IBM SPSS Inc., Armonk, NY, USA). A value of $\mathrm{p}<0.05$ was considered statistically significant.

\section{RESULTS}

\section{General characteristics of subjects}

General physical characteristics of the experimental and control groups were described in Table 1. Total 21 adults with CP (11 men and 10 women) consisted of 14 in the experimental group ( 7 men and 7 women) and 7 in the control group ( 4 men and 3 women). There were no significant differences in age, sex, height, weight, body mass index, systolic blood pressure, and diastolic blood pressure between the two groups of CP patients (Table 1).

Changes in the parameters of energy consumption test during treadmill walking

The results collected on a treadmill after treadmill walking exercise or conventional physical therapy was shown in Table 2. There were no significant changes in all the parameters, such as walking distance, time, velocity, $\mathrm{VO}_{2}$, $\mathrm{VCO}_{2}, \mathrm{VE}$, and $\mathrm{O}_{2}$ rate between the pre-treatment and post-treatment states (Table 2). However, energy expenditure per meter, $\mathrm{O}_{2}$ cost was significantly increased from $0.42 \pm 0.23$ to $0.58 \pm 0.38 \mathrm{~mL} / \mathrm{kg} \cdot \mathrm{m}$ after conventional physical therapy in the control group $(\mathrm{p}=0.046)$, suggestive of

Table 2. Changes in the parameters of energy consumption test during treadmill walking

\begin{tabular}{lccccc}
\hline & \multicolumn{2}{c}{ Experimental group $(\mathbf{n}=\mathbf{1 4})$} & & \multicolumn{2}{c}{ Control group (n=7) } \\
\cline { 2 - 3 } \cline { 5 - 6 } & Pre-treatment & Post-treatment & & Pre-treatment & Post-treatment \\
\hline Distance $(\mathrm{m})$ & $117.14 \pm 60.26$ & $120.71 \pm 58.50$ & & $156.43 \pm 70.63$ & $154.29 \pm 63.99$ \\
Time $(\mathrm{min})$ & $5.79 \pm 0.43$ & $5.93 \pm 0.27$ & & $5.57 \pm 1.13$ & $6.00 \pm 0.00$ \\
\hline Velocity $(\mathrm{m} / \mathrm{min})$ & $20.36 \pm 11.34$ & $20.71 \pm 11.30$ & & $27.86 \pm 10.22$ & $25.71 \pm 10.67$ \\
$\mathrm{VO}_{2}(\mathrm{~mL} / \mathrm{min})$ & $630.92 \pm 251.73$ & $595.38 \pm 222.20$ & & $558.97 \pm 263.53$ & $688.32 \pm 387.14$ \\
$\mathrm{VCO}_{2}(\mathrm{~mL} / \mathrm{min})$ & $543.58 \pm 283.68$ & $433.21 \pm 205.96$ & & $484.57 \pm 287.73$ & $544.99 \pm 246.86$ \\
$\mathrm{VE}(\mathrm{L} / \mathrm{min})$ & $18.55 \pm 6.91$ & $17.90 \pm 5.89$ & & $19.21 \pm 10.48$ & $20.70 \pm 8.34$ \\
$\mathrm{O}_{2}$ rate $(\mathrm{mL} / \mathrm{kg} \cdot \mathrm{min})$ & $11.49 \pm 3.31$ & $10.86 \pm 2.73$ & & $10.13 \pm 2.52$ & $12.02 \pm 3.10$ \\
$\mathrm{O}_{2}$ cost $(\mathrm{mL} / \mathrm{kg} \cdot \mathrm{m})$ & $0.69 \pm 0.29$ & $0.65 \pm 0.30$ & & $0.42 \pm 0.23$ & $0.58 \pm 0.38^{*}$ \\
\hline
\end{tabular}

Values are presented as mean \pm standard deviation.

$\mathrm{VO}_{2}$, oxygen uptake per minute; $\mathrm{VCO}_{2}$, carbon dioxide production amount per minute; $\mathrm{VE}$, respiratory volume per minute; $\mathrm{O}_{2}$ rate, oxygen consumption rate; $\mathrm{O}_{2}$ cost, oxygen consumption cost.

${ }^{*} \mathrm{p}<0.05$. 
an increase in energy expenditure with no improved gait efficiency during treadmill walking (Table 2).

Changes in the parameters of energy consumption test during overground walking

The results obtained during overground walking up to 6 minutes after the treadmill walking exercise or conventional physical therapy was described in Table 3. After the treadmill walking exercise, the experimental group showed that gait distance significantly increased from $151.29 \pm 91.79$ to $193.93 \pm 79.01 \mathrm{~m}$, and gait velocity increased from $28.09 \pm 14.29$ to $33.49 \pm 12.69 \mathrm{~m} / \mathrm{min}$ ( $\mathrm{p}=0.048$ each) (Table 3). Moreover, $\mathrm{O}_{2}$ cost was significantly de- creased from $0.56 \pm 0.36$ to $0.41 \pm 0.18 \mathrm{~mL} / \mathrm{kg} \cdot \mathrm{m}$ in the experimental group $(\mathrm{p}=0.03)$, suggestive of an improved gait efficiency post-treadmill walking exercise (Table 3). In contrast, there were no significant changes in the control group.

Comparison of energy expenditure parameters between experimental and control groups

The parameters of energy consumption test, such as the difference between post-treatment and pre-treatment states $(\Delta)$ in the $\mathrm{O}_{2}$ rate and the $\mathrm{O}_{2}$ cost, were then compared between experimental and control groups. As a result, there were no significant differences in the $\Delta \mathrm{O}_{2}$

Table 3. Changes in parameters of energy consumption test during overground walking

\begin{tabular}{lccccc}
\hline & \multicolumn{2}{c}{ Experimental group $(\mathbf{n}=\mathbf{1 4})$} & & \multicolumn{2}{c}{ Control group (n=7) } \\
\cline { 2 - 3 } \cline { 5 - 6 } & Pre-treatment & Post-treatment & & Pre-treatment & Post-treatment \\
\hline Distance $(\mathrm{m})$ & $151.29 \pm 91.79$ & $193.93 \pm 79.01^{*}$ & & $162.14 \pm 81.85$ & $180.71 \pm 61.40$ \\
Time $(\mathrm{min})$ & $5.14 \pm 1.23$ & $5.71 \pm 0.83$ & & $5.14 \pm 1.46$ & $5.74 \pm 0.69$ \\
Velocity $(\mathrm{m} / \mathrm{min})$ & $28.09 \pm 14.29$ & $33.49 \pm 12.69^{*}$ & & $29.88 \pm 9.96$ & $31.43 \pm 9.40$ \\
$\mathrm{VO}_{2}(\mathrm{~mL} / \mathrm{min})$ & $646.75 \pm 240.47$ & $656.70 \pm 283.11$ & & $640.67 \pm 415.05$ & $733.11 \pm 431.30$ \\
$\mathrm{VCO}_{2}(\mathrm{~mL} / \mathrm{min})$ & $516.98 \pm 212.56$ & $559.09 \pm 310.48$ & & $573.62 \pm 497.45$ & $597.89 \pm 336.90$ \\
$\mathrm{VE}(\mathrm{L} / \mathrm{min})$ & $18.96 \pm 6.31$ & $21.68 \pm 10.17$ & & $22.23 \pm 15.99$ & $22.96 \pm 11.77$ \\
$\mathrm{O}_{2}$ rate $(\mathrm{mL} / \mathrm{kg} \cdot \mathrm{min})$ & $11.74 \pm 2.53$ & $11.88 \pm 3.38$ & & $10.94 \pm 3.99$ & $12.36 \pm 3.81$ \\
$\mathrm{O}_{2}$ cost $(\mathrm{mL} / \mathrm{kg} \cdot \mathrm{m})$ & $0.56 \pm 0.36$ & $0.41 \pm 0.18^{*}$ & & $0.40 \pm 0.16$ & $0.43 \pm 0.21$ \\
\hline
\end{tabular}

Values are presented as mean \pm standard deviation.

$\mathrm{VO}_{2}$, oxygen uptake per minute; $\mathrm{VCO}_{2}$, carbon dioxide production amount per minute; VE, respiratory volume per minute; $\mathrm{O}_{2}$ rate, oxygen consumption rate; $\mathrm{O}_{2}$ cost, oxygen consumption cost.

${ }^{*} \mathrm{p}<0.05$.
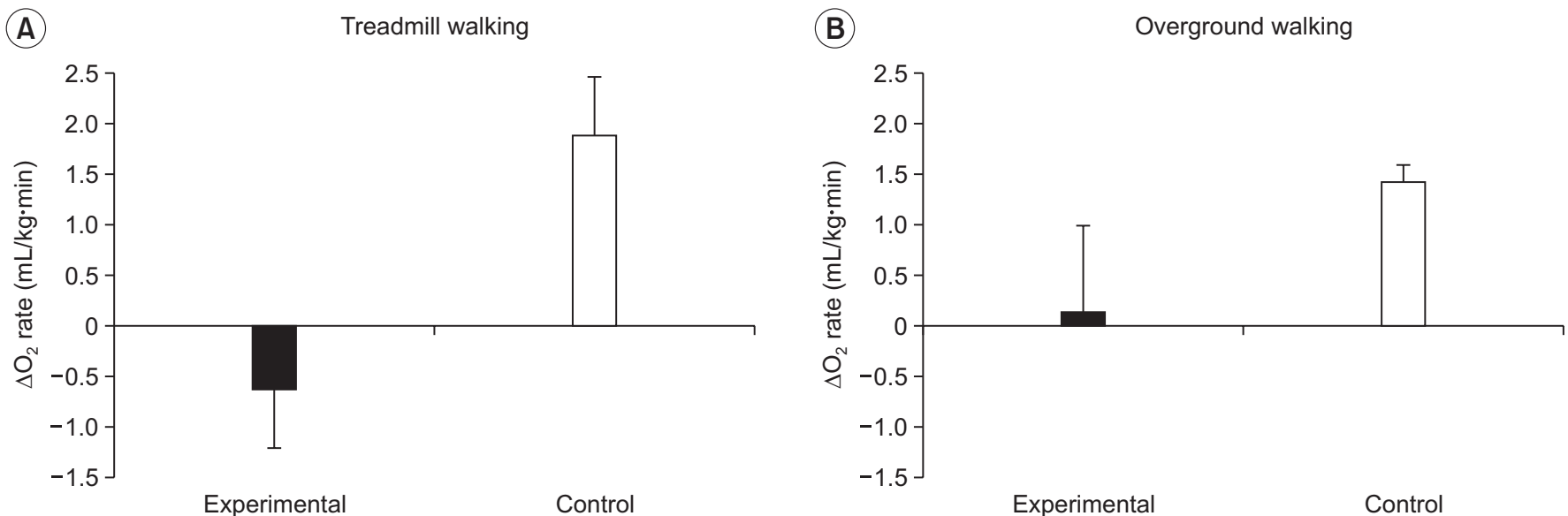

Fig. 1. Comparison of $\Delta \mathrm{O}_{2}$ rate between experimental and control groups. (A) Difference in post-treatment to pretreatment states $(\Delta)$ in the $\mathrm{O}_{2}$ rate $(\mathrm{mL} / \mathrm{kg} \cdot \mathrm{min})$ after treadmill training had no significant differences in the parameter during treadmill walking between experimental and control groups. (B) The experimental group did not show a significant improvement in the $\Delta \mathrm{O}_{2}$ rate during overground walking. 

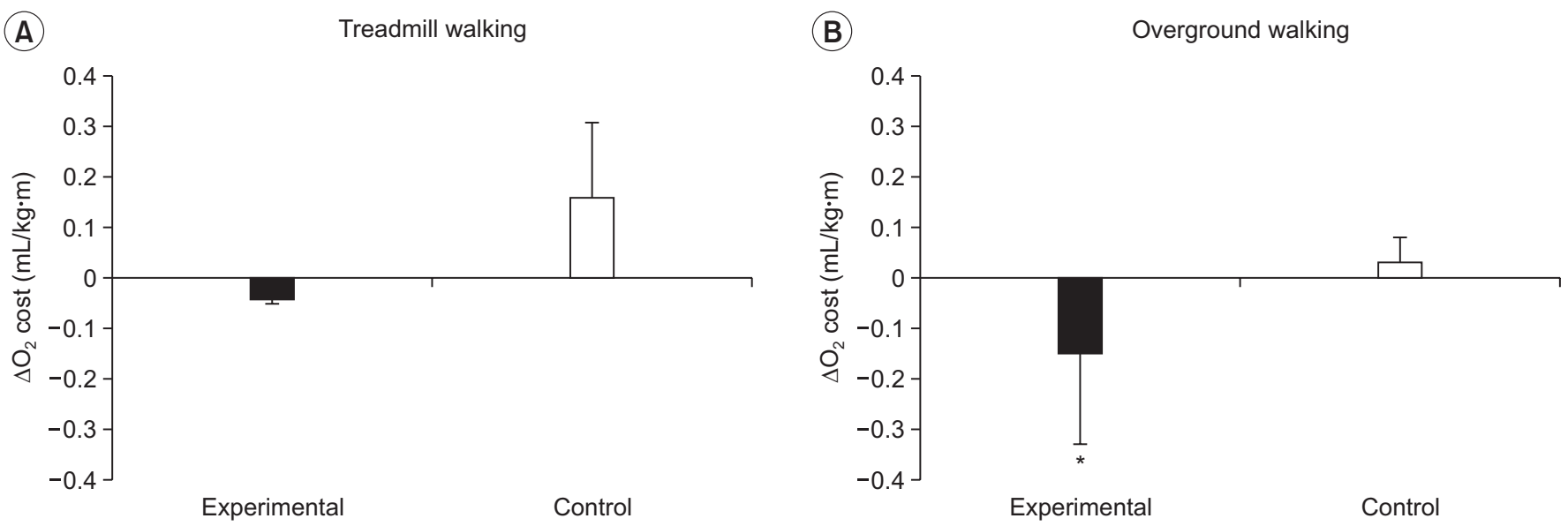

Fig. 2. Comparison of $\Delta \mathrm{O}_{2}$ cost between experimental and control groups. (A) Difference from post-treatment to pretreatment states $(\Delta)$ in the $\mathrm{O}_{2}$ cost $(\mathrm{mL} / \mathrm{kg} \cdot \mathrm{m})$ after treadmill training had no significant differences in the parameter during treadmill walking between experimental and control groups. (B) However, the experimental group showed significantly lower $\Delta \mathrm{O}_{2}$ cost, as compared with the control group during overground walking $\left({ }^{*} \mathrm{p}<0.05\right)$.

rate during both treadmill walking and overground walking between the two groups (Fig. 1A, B). However, the experimental group showed significantly lower $\Delta \mathrm{O}_{2}$ cost $(-0.15 \pm 0.18 \mathrm{~mL} / \mathrm{kg} \cdot \mathrm{m})$, as compared with the control group $(0.04 \pm 0.05 \mathrm{~mL} / \mathrm{kg} \cdot \mathrm{m})$ during overground walking ( $\mathrm{p}=0.04$ ) (Fig. 2B), although the $\Delta \mathrm{O}_{2}$ cost was not significantly different between the two groups during treadmill walking (Fig. 2A).

\section{DISCUSSION}

A previous study suggested that individuals with $\mathrm{CP}$ are less active and more sedentary than their able-bodied peers [12]. Children and adolescents with CP tend to participate in less structured and lower intensity physical activities. A vicious cycle of exposure to lower physical activities in persons with chronic illness or disability leads to further deconditioning and physical deterioration [13]. Children with $\mathrm{CP}$ at the age of 7 have already lowered $\mathrm{VO}_{2}$ peak values compared to typical developing peers. Therefore, early intervention for an active lifestyle might be beneficial for promoting physical fitness in CP patients [14].

Schindl et al. [15] reported the effects of body-weight supported treadmill walking exercise as a treatment method to improve the physical fitness in children with CP who were mostly non-ambulatory; their data demonstrate relevant improvements in motor abilities. Activity patterns shift towards aerobic activity in adulthood, making aerobic capacity more important with increasing age [14]. Therefore, there was a need for studying adult subjects in treatments to improve aerobic capacity and physical fitness, such as treadmill walking exercise. We focused on adult patients with $\mathrm{CP}$ who have ambulatory function.

Oxygen uptake measures may be the best method to quantify gait efficiency during functional walking. Bowen et al. [16] reported $\mathrm{O}_{2}$ cost as a more reliable measurement of energy expenditure than $\mathrm{VO}_{2}$ alone. $\mathrm{O}_{2}$ cost is determined by dividing $\mathrm{O}_{2}$ consumption by the walking speed $(\mathrm{mL} / \mathrm{kg} \cdot \mathrm{m})$ as an indicator of gait efficiency. Duffy et al. [17] demonstrated that the $\mathrm{O}_{2}$ cost was significantly higher in children with spina bifida and $\mathrm{CP}$ compared to healthy children. In this study, ambulatory CP patients in the experimental group increased their walking distance and gait velocity, leading to a decrease in the $\mathrm{O}_{2}$ cost during overground walking after treadmill walking exercise. It suggested that treadmill walking exercise characteristically improved the gait efficiency during overground walking.

When the $\mathrm{O}_{2}$ cost of walking on a treadmill with those of overground walking is compared after treadmill walking exercise, it is possible to clarify the gait efficiency of both conditions. Little is known about the differences in the $\mathrm{O}_{2}$ cost on a treadmill versus overground walking; hence, a study must be conducted to determine the more efficient $\mathrm{O}_{2}$ cost in order to verify gait efficiency for treadmill training. As a result, the $\mathrm{O}_{2}$ cost measured during over- 
ground walking was significantly improved, as compared with the $\mathrm{O}_{2}$ cost during treadmill walking, suggestive of improved gait efficiency during overground walking in ambulatory patients.

The effects of treadmill training on overground walking speed have been reported [2]. On the other hand, preferred walking speed determined on a treadmill is slower compared to overground, leading to higher $\mathrm{O}_{2}$ cost on the treadmill, although the treadmill is often used to simulate overground walking [9]. Recent evidence indicates that when the direction of walking is the same for the 2 conditions, similar neural networks may be involved; however, when on the treadmill, subjects do not move through space as they do during overground walking. These contextual differences arise from the lack of visual and somatosensory information during treadmill walking. It is possible that these identifiable differences in task context are sufficient to transfer from the treadmill walking exercise to a more natural overground walking environment with more efficient gait [18].

In conclusion, treadmill walking exercise improved gait efficiency in adults with CP. It has been associated with decreased energy expenditure measured by $\mathrm{O}_{2}$ cost during overground walking. Therefore, treadmill walking exercise can be an important method for gait training in ambulatory CP patients who have higher energy expenditure and lower gait efficiency.

\section{CONFLICT OF INTEREST}

No potential conflict of interest relevant to this article was reported.

\section{ACKNOWLEDGMENTS}

The work was supported by the National Research Foundation (NRF-2010-0020408, 2014R1A2A1A11052042) funded by the Ministry of Education, Science and Technology, Republic of Korea.

\section{REFERENCES}

1. Bax M, Goldstein M, Rosenbaum P, Leviton A, Paneth N, Dan B, et al. Proposed definition and classification of cerebral palsy, April 2005. Dev Med Child Neurol 2005;47:571-6.
2. Willoughby KL, Dodd KJ, Shields N, Foley S. Efficacy of partial body weight-supported treadmill training compared with overground walking practice for children with cerebral palsy: a randomized controlled trial. Arch Phys Med Rehabil 2010;91:333-9.

3. Provost B, Dieruf K, Burtner PA, Phillips JP, BernitskyBeddingfield A, Sullivan KJ, et al. Endurance and gait in children with cerebral palsy after intensive body weight-supported treadmill training. Pediatr Phys Ther 2007;19:2-10.

4. Dodd KJ, Foley S. Partial body-weight-supported treadmill training can improve walking in children with cerebral palsy: a clinical controlled trial. Dev Med Child Neurol 2007;49:101-5.

5. Mattern-Baxter K. Effects of partial body weight supported treadmill training on children with cerebral palsy. Pediatr Phys Ther 2009;21:12-22.

6. Sullivan KJ, Knowlton BJ, Dobkin BH. Step training with body weight support: effect of treadmill speed and practice paradigms on poststroke locomotor recovery. Arch Phys Med Rehabil 2002;83:683-91.

7. Moseley AM, Stark A, Cameron ID, Pollock A. Treadmill training and body weight support for walking after stroke. Cochrane Database Syst Rev 2005;(4):CD002840.

8. Norman JF, Bossman S, Gardner P, Moen C. Comparison of the energy expenditure index and oxygen consumption index during self-paced walking in children with spastic diplegia cerebral palsy and children without physical disabilities. Pediatr Phys Ther 2004;16:206-11.

9. Dal U, Erdogan T, Resitoglu B, Beydagi H. Determination of preferred walking speed on treadmill may lead to high oxygen cost on treadmill walking. Gait Posture 2010;31:366-9.

10. Kim SJ, Kwak EE, Park ES, Cho SR. Differential effects of rhythmic auditory stimulation and neurodevelopmental treatment/Bobath on gait patterns in adults with cerebral palsy: a randomized controlled trial. Clin Rehabil 2012;26:904-14.

11. Lipkin DP, Scriven AJ, Crake T, Poole-Wilson PA. Six minute walking test for assessing exercise capacity in chronic heart failure. Br Med J (Clin Res Ed) 1986; 292:653-5.

12. Maher CA, Williams MT, Olds T, Lane AE. Physical and sedentary activity in adolescents with cerebral 
palsy. Dev Med Child Neurol 2007;49:450-7.

13. Durstine JL, Painter P, Franklin BA, Morgan D, Pitetti $\mathrm{KH}$, Roberts SO. Physical activity for the chronically ill and disabled. Sports Med 2000;30:207-19.

14. Verschuren O, Takken T. Aerobic capacity in children and adolescents with cerebral palsy. Res Dev Disabil 2010;31:1352-7.

15. Schindl MR, Forstner C, Kern H, Hesse S. Treadmill training with partial body weight support in nonambulatory patients with cerebral palsy. Arch Phys Med Rehabil 2000;81:301-6.

16. Bowen TR, Miller F, Mackenzie W. Comparison of oxygen consumption measurements in children with cerebral palsy to children with muscular dystrophy. J Pediatr Orthop 1999;19:133-6.

17. Duffy CM, Hill AE, Cosgrove AP, Corry IS, Graham HK. Energy consumption in children with spina bifida and cerebral palsy: a comparative study. Dev Med Child Neurol 1996;38:238-43.

18. Reisman DS, Wityk R, Silver K, Bastian AJ. Split-belt treadmill adaptation transfers to overground walking in persons poststroke. Neurorehabil Neural Repair 2009;23:735-44. 